JOURNAL DE PHYSIQUE IV

Colloque C7, supplément au Journal de Physique III, Volume 4, juillet 1994

\title{
The analysis of error in trace gas concentration measurements by intermodulated photoacoustic stark spectroscopy (IMPASS)
}

\author{
T. Groot, D. Bicanic, M. van Montfort*, P. Torfs**, R. Braun and K. van Asselt \\ Laser Photoacoustic Laboratory, Department of Agricultural Engineering and Physics, Agricultural \\ University, Bomenweg 4, 6703 HD Wageningen, The Netherlands \\ * Department of Mathematics, Dreijenlaan 4, Agricultural University, 6703 HA Wageningen, The \\ Netherlands \\ ** Department of Water Resources, Agricultural University, de Nieuwlanden, Nieuwe Kanaal 11, \\ 6709 PA Wageningen, The Netherlands
}

\begin{abstract}
Data collected in an actual IMPASS experiment with a static gaseous mixture (methyl fluoride/ethylene/nitrogen) of preselected concentrations were analyzed with respect to uni(bi)modality, symmetry, skeweness and kurtosis. Bimodal probability distribution function is significantly different from Gaussian suggesting the presence of "mixed" data in the measurements; the potential cause for this phenomenon is discussed.
\end{abstract}

\section{INTRODUCTION}

Photoacoustic (PA) spectroscopy with intense lasers is often employed for sensitive concentration measurements of gases under variety of conditions. In a PA experiment, the unknown concentration (c) of gas confined in an acoustic cell (responsivity $R$ ) is found from $c=S /(R P \sigma)$ where $S$ represents the magnitude of the microphone signal, $\mathrm{P}$ is the excitation power (at a given wavelength) and $\sigma$ is the absorption coefficient. The concept of IMPASS (InterModulated PA Stark Spectroscopy), a sophisticated version of PA (it comprises the simultaneous modulation of incident laser power and of electric field applied to the gas) was recently introduced [1] to eliminate effects of interfering gases and parasitic signals originating from heated cell windows and chopper noise. In IMPASS mode, the signal is proportional to $\mathrm{P}, \mathrm{R}$, and $\mathrm{c}$ of the gas, but also to the modulation depth of $\sigma$ (related to applied electric field $E_{S}$ ). Despite the fact that much work is still going on and reports on new measurements accumulate, there are comparatively little reports in scientific literature on the reproducibility achievable by PA and IMPASS techniques.

At least two numbers ( $\mathrm{x}$ and $\Delta \mathrm{x}$ ) are required if an experiment is to give a result and a measure of its reliability. By adopting the notation $(x \pm \Delta x)$ for the result of a measurement, one tacitly assumes that error is additive and symmetric (which is characteristic for Gaussian distribution). Likewise, when stating that $68 \%$ (or $95 \%)$ of measured values will be found between $x \pm \Delta x(x \pm 2 \Delta x)$, one tacitly assumes that the error is Gaussian.

The objective of this paper is to make experimentalist aware of a need to perform an error analysis; an illustrative example is given. Overlooking the extent of error might lead to errors when interpreting data. After introducing some basic theoretical considerations and giving a description of a set-up, an error analysis is applied to data collected in a realistic IMPASS study. The aim of our 
investigation was the detection of $\mathrm{CH}_{3} \mathrm{~F}$ in a matrix of abundant $\mathrm{C}_{2} \mathrm{H}_{4}$ and $\mathrm{N}_{2}$ gases. The reason for selecting such composition was to demonstrate immunity of IMPASS to spectral intereferences $\left(\mathrm{C}_{2} \mathrm{H}_{4}\right.$ has large $\sigma$ but a zero dipole moment which is opposite for the polar $\mathrm{CH}_{3} \mathrm{~F}$ gas).

\section{SOME THEORETICAL CONSIDERATIONS}

As already mentioned, it is important to find out whether or not data distribution is Gaussian. A quick answer to this is provided by a "Gauss plot" [2]. Basically, it implies plotting inverse empirical cumulative distribution function versus the inverse cumulative Gaussian. If, and only if, distribution of data is a true Gaussian, the plot will be a straight line. In such a case slope and intercept (y-axis) can be used to estimate standard deviation and the mean value, respectively.

Given a certain probability distribution function, there are additional approaches capable of finding out in which way it does depart from a Gaussian. These are based on inspection of data for occurence of features characteristic for Gaussian. The unimodality (probability distribution function displays a single peak) is a first characteristic and essentially different from bimodality observed when abusively "mixed" data are collected under two different experimental conditions. Should the probability distribution function suggest the presence of bimodality, the experimentalist is urged to identify a cause for its occurence and if possible remove it. In order to check whether or not data are bimodal, a "chisquare test" on the likelihood functions can be utilized to test between a unimodal Gaussian and a bimodal generated by mixing two Gaussian distributions with different means and variances [3]. Second relevant property is the symmetry of the Gaussian that can be quantified by the skewness factor (i.e. third moment about the mean, normalized by third power of standard deviation)[2]. If data are distributed symmetrically (Gaussian) skewness is zero. For positive skewness, more data are found to the right of the mean (compared to Gaussian having same mean and standard deviation). Likewise, negative skewness indicates the incidence of fewer data. The concept of kurtosis (calculated by dividing fourth moment about the mean by fourth power of standard deviation) can also be used to characterize data distributions [2]. Zero kurtosis is assigned to data exhibiting a Gaussian distribution. When kurtosis is positive (negative), distribution has significantly higher (lower) tails then in corresponding Gaussian case. In a unimodal distribution, a sharp (flattened) peak is associated with a positive (negative) kurtosis.

If after completing above mathematical analysis it appears that data are unimodal but not Gaussian, an attempt can be made to transform the original set of data (e.g. to take logarithms of measured values instead of measured values themselves) to produce a more Gaussian like distribution.

\section{EXPERIMENTAL}

Typical IMPASS set up (Fig. 1) includes a $\mathrm{CO}_{2}$ laser (E), modulator (B), Stark PA cell (A) (provided with electrodes and modulator) and lens (C). The PA cell, coupled to gas supply and pump can be operated in flow-through and sealed off modus. A device (D) consisting of four cubes (each provided with a plane mirror at $45^{\circ}$ ) put together enables one to rotate the plane of the laser E field [4]. For detection of some gases the direction of the laser $E$ field perpendicular to the Stark electric field $E_{\mathrm{S}}$ is preferred. The microphone signal is fed to the lock-in amplifier. A pyroelectric power detector (11) was used behind the PA cell, in order to normalize the signals especially for weakly absorbing samples. A Ge plate (12) reflects a fraction of the laser radiation into a spectrum analyzer (F in Fig. 1) in order to monitor the laser wavelength.

The measurements carried out to estimate a reproducibility of IMPASS were performed with a (self made) mixture of $11 \mathrm{ppmv}$ of $\mathrm{CH}_{3} \mathrm{~F}$ in $68 \mathrm{ppbv}$ of ethylene (remaining fraction is nitrogen). The laser ( 3 Watt) was tuned to $9 \mathrm{P}(18)$ transition corresponding to a wavelength of maximal absorption for $\mathrm{CH}_{3} \mathrm{~F}\left(13 \mathrm{~atm}^{-1} \mathrm{~cm}^{-1}\right)$. The cell was loaded to $200 \mathrm{mbar}$; this value is an optimum in terms of 

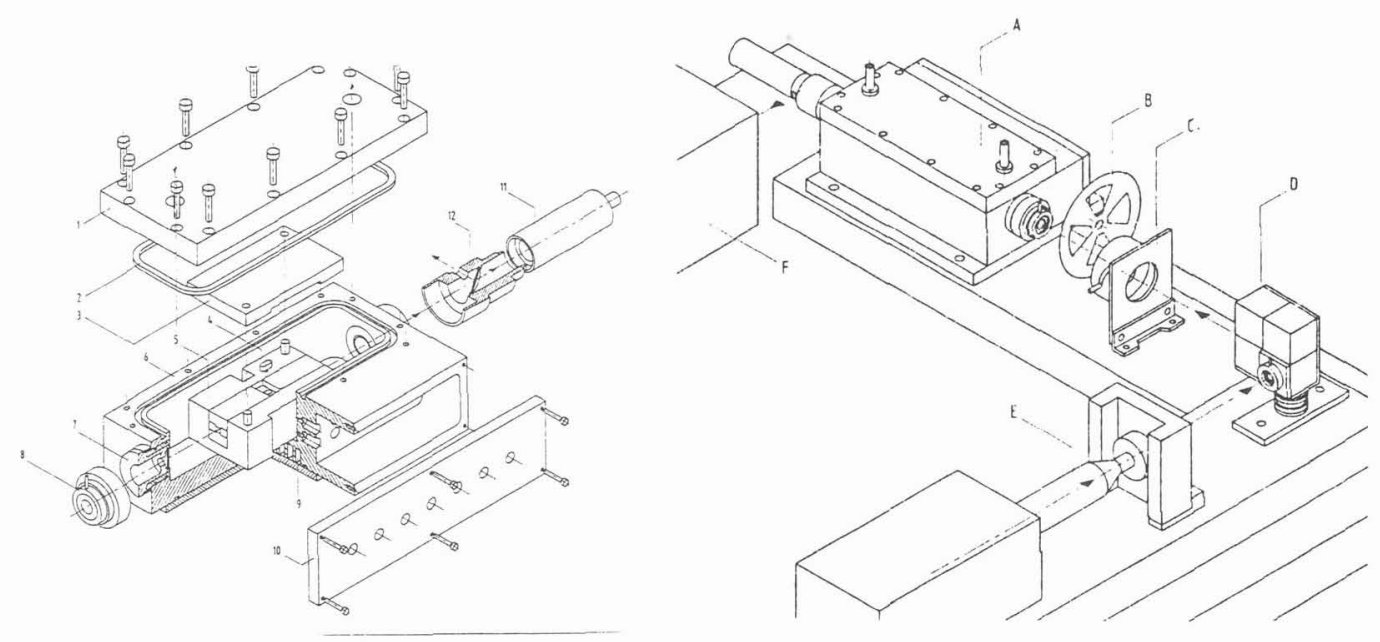

Fig. 1 The experimental arrangement (right) and PA cell with detector (left) for gas concentration measurements by the IMPASS method.

microphone sensitivity, linewidth and prevention of electrical breakdown in the cell. The PA cell was used with a static gas filling. The electric field applied to the Stark plates (separated by $5 \mathrm{~mm}$ ) was typically $4.5 \mathrm{kV} / \mathrm{cm}$. The modulation frequencies of the laser and electric field were $170 \mathrm{~Hz}$ and 1780 $\mathrm{Hz}$, respectively. The difference frequency $(1610 \mathrm{~Hz})$ equals that of the cell's first longitudinal acoustic resonance and is used as a reference frequency for the lock-in amplifier.

The microphone signal $\mathbf{S}$, laser power $\mathbf{P}$ (behind the cell and beam splitter), cell pressure and high voltage applied to the Stark plates were recorded automatically (Hewlett Packard data logger 3497 A). These values were used to compute normalized IMPASS signals. Approximately one minute was needed to complete a single scan. Four such successive scans (termed "series") were averaged. In total, 48 series were performed on different days using fresh gas fillings.

\section{RESULTS AND DISCUSSION}

Mean values obtained in each one of the 48 series were averaged giving an overall mean of 2.83 $\mu \mathrm{V} /\left(\mathrm{cm}^{-1} \mathrm{~atm}^{-1}\right.$ Watt). The Gauss plot (shown in Fig. 2) of measured data is nearly a straight line except for low $\mathrm{x}$-values and thus cannot be classified as Gaussian. Standard deviation is $0.18 \mu \mathrm{V} /\left(\mathrm{cm}^{-1} \mathrm{~atm}\right.$ ${ }^{1}$ Watt) when considering eight lowest values (where a slope is steeper) and $0.11 \mu \mathrm{V} /\left(\mathrm{cm}^{-1} \mathrm{~atm}^{-1} \mathrm{Watt}\right)$ (with remaining 40 values).

The application of the uni(bi)modality test reveals that distribution is bimodal (difference in likelihood values exceeds $\chi^{2}$ ). In order to get a better insight to what it concerns the resemblance to Gaussian, skewness and kurtosis were calculated. The skewness coefficient is -1.43 (with a low $5 \%$ point of -0.71 ) so that the mean is shifted towards low values of normal signals. The coefficient of kurtosis is 3.04 (with a lower $5 \%$ decision point of 0.99 ) indicating occurence of high tails.

Summarizing, statistical analysis of data collected in a IMPASS measurement shows that they lack all characteristics of Gaussian distribution and hence expressing the outcome of measurement as $(\mathrm{x} \pm \Delta \mathrm{x})$ becomes questionable. Moreover, bimodality suggests that some "mixing" of experimental data apparently has taken place. 


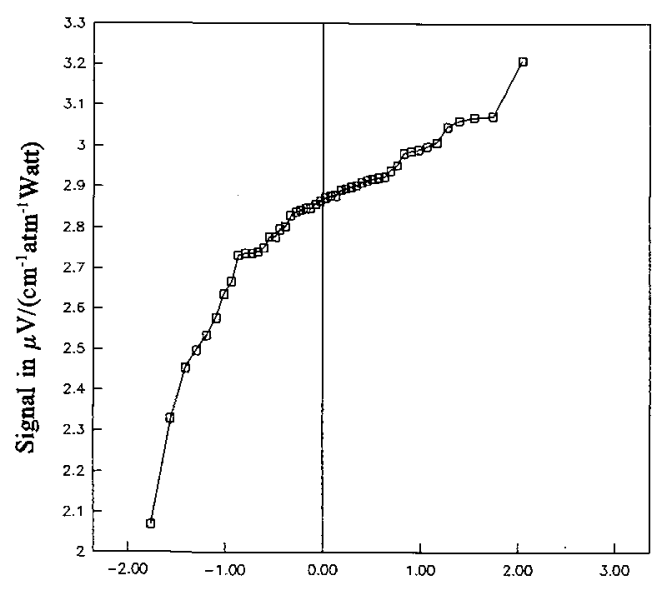

Fig. 2 The "Gauss" plot of measured data.

Basically two possible sources of error have to be considered briefly. First, the gas processing, which is known to be highly susceptible to errors especially when working in the trace gas regime. For example memory effects [1] or different sticking probabilities for different components in the mixture at different materials may result in large discrepancies between sampled (real) gas concentration and the one finally reaching the cell. Despite the possibly systematic character of this error it cannot hold for our observation.

Another interesting phenomenon was observed during an experiment recently performed with the same experimental set-up. With the Ge disc used as a beam splitter no positive correlation between power reaching the pyrodetector and incident laser power was observed. This might be attributed to the effect of multiple interference within the beam splitter itself that affects its transmission characteristic. More precisely, transmission of the beam splitter (thickness d, refractive index $\mathbf{n}$ ) is a function of the angle of incidence, wavelength, and optical thickness (nd) which itself depends on temperature and optical absorption in the device. As a result, the pyroelectric signal (in the way as it is measured in the present set-up) is no longer representative of the laser power within the PA cell. Since power enters the basic PA equation for computing the unknown gas concentration, values obtained in such a way will not only lead to a non-Gaussian distribution but also to an erroneous interpretation of the concentration measurement. For this reason one must be cautious using beam splitters for monitoring power or wavelength. To counteract this effect the beam splitter behind the cell (Fig. 1,(12)) was removed and instead the plane mirror (not shown in Fig. 1) oriented at $45^{\circ}$ (with respect to optical axis and positioned in front of the PA cell) ocassionally inserted in the radiation path of the laser to control the identitiy of laser emission.

\section{REFERENCES}

[1] Sauren J.J.A.M., "Ammonia monitor based on intermodulated $\mathrm{CO}_{2}$ laser photoacoustic Stark spectroscopy", Ph.D. Thesis, Agricultural University, Wageningen, The Netherlands (1992).

[2] Snedecor G.W., Cochran W.G., Statistical methods, The Iowa State University Press, Ames, Iowa, USA (1990).

[3] Braak ter, C.J., Priv. Comm., GLW-DLO, Wageningen, The Netherlands (1993).

[4] Olafsson A., University of Reykjavik, Iceland, Priv. Comm. (1993). 